\title{
Minimização dos impactos ambientais causados pelos resíduos da indústria têxtil:
}

\section{Uma perspectiva prática}

\author{
Minimizing environmental impacts caused by textile waste: A practical perspective \\ Minimizar los impactos ambientales causados por los desechos textiles: Una perspectiva práctica
}

Recebido: 20/01/2021 | Revisado: 23/01/2021 | Aceito: 09/02/2021 | Publicado: 20/02/2021

\author{
Ana Carolina Dias de Albuquerque \\ ORCID: https://orcid.org/0000-0003-1620-5708 \\ Universidade Cesumar, Brasil \\ E-mail: acdalbuquerque@hotmail.com \\ Marla Corso \\ ORCID: https://orcid.org/0000-0001-5168-5407 \\ Universidade Positivo, Brasil \\ E-mail: marlacorso@ hotmail.com \\ Lilian Keylla Berto \\ ORCID: https://orcid.org/0000-0003-0343-9433 \\ Universidade Cesumar, Brasil \\ E-mail: lilianberto_engenharia@hotmail.com \\ Flávia Aparecida Reitz Cardoso \\ ORCID: https://orcid.org/0000-0002-0432-9191 \\ Universidade Tecnológica Federal do Paraná, Brasil \\ E-mail: flaviareitz@gmail.com \\ Eliane Carvalho de Vasconcelos \\ ORCID: https://orcid.org/0000-0002-5923-0834 \\ Universidade Positivo, Brasil \\ E-mail: evasconcelos@up.edu.br \\ Silvia Lucia Favaro \\ ORCID: https://orcid.org/0000-0002-3963-1892 \\ Universidade Estadual de Maringá, Brasil \\ E-mail: slfavaro@hotmail.com \\ Luciana Cristina Soto Herek Rezende \\ ORCID: https://orcid.org/0000-0001-9677-4139 \\ Universidade Cesumar, Brasil \\ Instituto Cesumar de Ciência, Tecnologia e Inovação, Brasil \\ E-mail: luciana.rezende@unicesumar.edu.br
}

\section{Resumo}

A preocupação ambiental com os resíduos gerados pelos processos produtivos da indústria têxtil fomentou o desenvolvimento de pesquisas na área. O desenvolvimento de novos materiais a partir dos resíduos gerados pela mesma é uma alternativa para destinar todo o material acumulado, agregando assim uma nova aplicação. Tendo também o setor construtivo um papel importante em relação à degradação ambiental no meio urbano, a busca por materiais que visem a sustentabilidade no setor é crescente, demandando diversas pesquisas que buscam o desenvolvimento de novos materiais para o setor. Face a estas considerações, buscou-se desenvolver um material alternativo a partir de resíduos de poliéster incorporados em uma matriz polimérica reciclada. Os materiais obtidos foram caracterizados por Granulometria e MEV, possibilitando identificar as suas propriedades e definir qual a melhor metodologia a ser empregada para confecção dos corpos de prova. Em seguida foram preparados materiais compósitos utilizando diferentes incorporações de poliéster $(10 \%, 20 \%, 30 \%, 40 \%$ e $50 \%)$ em relação à matriz de polipropileno reciclado. Com as análises dos ensaios de tração foi possível determinar que os melhores resultados obtidos ocorreram considerando a incorporação de $16,5 \%$ de fibra na matriz de polipropileno reciclado, dado este comprovado por ensaios de tração e impacto posteriores.

Palavras-chave: Compósitos; Matriz polimérica; Poliéster; Polipropileno reciclado; Sustentabilidade.

\begin{abstract}
The environmental concern with the residues generated by the textile industry's productive processes encouraged the development of research in the area. The development of new materials from the waste generated by it is an alternative to dispose of all the accumulated material, thus adding a new application. As the construction sector also plays an important role in relation to environmental degradation in the urban environment, the search for materials aimed at sustainability in the sector is growing, requiring several researches that seek the development of new materials for the sector. In view of these considerations, it was sought to develop an alternative material from
\end{abstract}


polyester residues incorporated in a recycled polymer matrix. The obtained materials were characterized by Granulometry and SEM, making it possible to identify their properties and define which is the best methodology to be used for making the specimens. Then composite materials were prepared using different polyester incorporations $(10 \%, 20 \%, 30 \%, 40 \%$ and $50 \%)$ in relation to the recycled polypropylene matrix. With the analysis of the tensile tests it was possible to determine that the best results obtained occurred considering the incorporation of $16.5 \%$ of fiber in the recycled polypropylene matrix, given this confirmed by subsequent tensile and impact tests.

Keywords: Composites; Polymeric matrix; Polyester; Recycled polypropylene; Sustainability.

\section{Resumen}

La preocupación ambiental con los residuos generados por los procesos productivos de la industria textil incentivó el desarrollo de investigaciones en la zona. El desarrollo de nuevos materiales a partir de los residuos que genera es una alternativa para disponer de todo el material acumulado, agregando así una nueva aplicación. Como el sector de la construcción también juega un papel importante en relación a la degradación ambiental en el medio urbano, la búsqueda de materiales orientados a la sustentabilidad en el sector es creciente, requiriendo varias investigaciones que buscan el desarrollo de nuevos materiales para el sector. En vista de estas consideraciones, se buscó desarrollar un material alternativo a partir de residuos de poliéster incorporados en una matriz de polímero reciclado. Los materiales obtenidos se caracterizaron mediante Granulometría y SEM, lo que permitió identificar sus propiedades y definir cuál es la mejor metodología a utilizar para la elaboración de las probetas. Posteriormente se prepararon materiales compuestos utilizando diferentes incorporaciones de poliéster $(10 \%, 20 \%, 30 \%, 40 \%$ y $50 \%)$ en relación a la matriz de polipropileno reciclado. Con el análisis de las pruebas de tracción se pudo determinar que los mejores resultados obtenidos se dieron considerando la incorporación de 16,5\% de fibra en la matriz de polipropileno reciclado, dado esto confirmado por posteriores pruebas de tracción e impacto.

Palabras clave: Composicion; Matriz polimérica; Poliéster; Polipropileno reciclado; Sustentabilidad.

\section{Introdução}

O conceito de desenvolvimento sustentável passou por diversas modificações desde a sua criação, fato resultado da conscientização dos problemas globais crescentes relacionados aos aspectos ambientais, sociais e econômicos. Não obstante à pobreza, desigualdade e preocupações com um futuro saudável para a humanidade em diferentes escalas todos têm sido reconhecidos como um grande desafio mundial (Djonú et al., 2018; Szopik-depczynska et al., 2018).

O crescimento populacional vinculado ao desenvolvimento dentro do processo de industrialização tem sido um dos fatores responsáveis, pois contribui para a geração de resíduos e degradação ambiental (Amorim et al., 2009; Bedekar et al., 2016; DIssanayake et al., 2018). A maior parte dos resíduos gerados não são reutilizados ou reciclados, de forma que o gerenciamento de resíduos é uma necessidade atual (Zeller et al., 2019).

O gerenciamento de resíduos é fundamental para o desenvolvimento sustentável no meio urbano, podendo ser de origem orgânica ou inorgânica, variando assim a sua destinação. Dentre os resíduos inorgânicos, o setor construtivo é o detentor do maior volume, causando grande impacto ambiental (Carpio et. al., 2016; Nielfa et al., 2015; Silva et al., 2019).

A demanda por recursos naturais no setor construtivo contribui para as emissões de $\mathrm{CO}_{2}$, intensificando o aquecimento global. Além disso, o grande volume de resíduos gerados por esse setor chamou a atenção dos pesquisadores para a criação de materiais que reaproveitem os resíduos, pois é uma forma de contribuir com a proteção ambiental e, por conseguinte, com o desenvolvimento sustentável (Aciu et al., 2018; Martin e Perry, 2019; Xu et al., 2019).

Uma das alternativas para mitigação deste impacto ambiental é a incorporação de diferentes resíduos em uma matriz polimérica, possibilitando a otimização das propriedades mecânicas dos materiais compósitos originados. Estes materiais são reconhecidos como adequados para construção civil visando benefícios ambientais, econômicos e sociais (Echeverria et al., 2019; Saccani et al. 2019; Sharma et al., 2018).

Dentre os compósitos, de forma geral destacam-se os que possuem matriz polimérica, em especial de polipropileno. Este é considerado um dos termoplásticos mais importantes devido ao seu baixo custo de fabricação, fácil processamento e por suas propriedades físicas, químicas e mecânicas relevantes (Albach et al., 2018). 
Os resíduos plásticos, quando dispostos na natureza de forma inadequada, tanto pela falta de gestão quanto pelo crescimento contínuo de sua utilização, tornam-se um risco para a saúde humana e ambiental (Karbalae et al., 2018; Ogunola et al., 2018). E devido sua crescente demanda e vasta utilização torna-se de grande valia para uso no cotidiano, contribuindo diretamente para a degradação ambiental. Face a estas considerações, existem diversas pesquisas que buscam solucionar esta questão ao incorporar o polipropileno como matéria-prima para o desenvolvimento de novos materiais (Aygörmez et al., 2020; Klapiszewski et al., 2019).

Com isso, há a possibilidade de melhoria das propriedades mecânicas da matriz de polipropileno por meio da incorporação de um elemento de reforço, sendo comumente empregada a utilização de fibras (Obaid et al., 2018). A origem das fibras pode ser natural ou química (sintética ou artificial), e quando categorizada como química contribui para a minimização da poluição ambiental e por esse motivo os seus resíduos tem tido ampla utilização (Qin et al., 2019).

As fibras sintéticas são advindas do petróleo e, por consequência, são fontes poluidoras (Dris et al., 2018). Um exemplo são as fibras de poliéster, constituídas por polietileno tereftalato (PET), possuindo propriedades que permitem facilidade de modificações físicas e químicas, agindo como um elemento de reforço para aumento das propriedades mecânicas dos compósitos (Militký, 2018).

Além disso, a incorporação do poliéster é tida como uma forma de reduzir o grande volume de resíduos têxteis gerados pelo setor têxtil. O descarte e o gerenciamento destes resíduos são um aspecto de grande preocupação ambiental (Haslinger et al., 2019; HU et al., 2018).

Diversos setores geram resíduos de poliéster, sendo que o principal poluidor é a indústria da moda, que desde o surgimento do fast fashion, um estímulo constante ao consumismo de uma população em pleno crescimento, contribuíram drasticamente para o aumento da poluição (Haslinger et al., 2019; Wanassi et al. 2017).

Desse modo, a sustentabilidade deve direcionar o processo de desenvolvimento das cidades atrelado aos Objetivos de Desenvolvimento Sustentável (ODS). Os ODS foram criados como um plano de ação que aborda aspectos para facilitar e direcionar esta iniciativa em conjunto com o governo, a sociedade civil, o setor privado e a comunidade científica (Costa, 2018).

Diante do exposto, este trabalho investigou a viabilidade de incorporar fibras de poliéster à matriz polimérica de polipropileno reciclado, pelo processo de injeção, para aplicação em elementos construtivos de revestimento.

\section{Metodologia}

Este estudo se caracteriza como uma pesquisa quantitativa, tendo como objetivo caracterizar o desenvolvimento de um novo material buscando seu melhor desempenho. A presente metodologia envolve levantamento bibliográfico, caracterização da metodologia e a sua possível replicação (Pereira A.S. et al., 2018).

\subsection{Obtenção e caracterização das matérias-primas}

O polipropileno reciclado (PPR) e a fibra têxtil (FP), composta por 100\% de poliéster, utilizados neste trabalho, foram obtidos em indústrias situadas na região de Maringá-PR/Brasil, de plástico e têxtil, respectivamente. Para o PPR foram obtidos quinze quilos de material na forma de grânulos e para a FP três quilos na forma de tecido.

A FP passou pelo processo de moagem com a duração de 180 segundos, com o material sendo disposto no bocal de um moinho da marca SEIBT modelo 250 LRX.

Os materiais obtidos, PPR e FP, foram analisados com relação à análise granulométrica por meio de peneiras com aberturas de $600 \mu \mathrm{m}$ à $9,5 \mathrm{~mm}$ e a morfologia foi realizada em um equipamento QUANTA 250 da marca FEI. 


\subsection{Preparo e confecção dos corpos de prova}

Para todas as composições dos corpos de prova (CPs), exceto para os CPs de referência, após a adição de FP ao PPR nas diferentes proporções (Tabela 1), os materiais foram triturados, também no moinho.

Os materiais foram dispostos em seis composições, com a matriz de polipropileno e com a incorporação de fibra, designadas como FP 0 , significa que contém $100 \%$ PPR e $0 \%$ de FP, seguindo essa determinação para as demais composições: FP 10, FP20, FP30, FP40 e FP50, proporções apresentadas na Tabela 1. Para cada proporção dos CPs, o PPR e a FP foram pesados separadamente em uma balança da marca Triunfo modelo DST 30/C-DM, de forma que cada composição foi armazenada em vasilhames plásticos.

Tabela 1 - Velocidade utilizada para a realização da análise de tração (ASTM D638).

\begin{tabular}{c|c|c|c}
\hline $\begin{array}{c}\text { Corpos de Prova } \\
\text { (CPs) }\end{array}$ & $\begin{array}{c}\text { Polipropileno } \\
\text { (PPR) }\end{array}$ & $\begin{array}{c}\text { Fibra de Poliéster } \\
\text { (FP) }\end{array}$ & $\begin{array}{c}\text { Velocidade de Ensaio } \\
\text { (mm/min) }\end{array}$ \\
\hline FP 0 & 100 & 0 & 50 \\
\hline FP 10 & 90 & 10 & 40 \\
\hline FP 20 & 80 & 20 & 30 \\
\hline FP 30 & 70 & 30 & 10 \\
\hline FP 40 & 60 & 40 & 5 \\
\hline FP 50 & 50 & 50 & 5 \\
\hline
\end{tabular}

Fonte: Autores (2020).

Os CPs foram confeccionados por meio da técnica de injeção. Foi utilizada uma injetora da marca ROMI modelo PRIMAX 100R, com temperatura do canhão de $215^{\circ} \mathrm{C}$, temperatura de molde $180^{\circ} \mathrm{C}$, pressão de injeção de 445 bar, tempo de injeção de 30s, pressão e recalque de 300 bar e tempo de recalque de 30s. Foram consideradas as normativas para os ensaios de tração e impacto ASTM D638 e ASTM D256, respectivamente.

\subsection{Caracterização dos corpos de prova}

Os corpos de prova confeccionados foram analisados com relação à morfologia por microscopia eletrônica de varredura em equipamento QUANTA 250 da marca FEI.

O ensaio de tração foi realizado em uma máquina universal de ensaios EMIC DL10000, submetido a uma célula de carga de $5 \mathrm{kN}$ com velocidade (mm/min): 50 para FP 0; 40 para FP 10; 30 para FP 20; 10 para FP 30; 5 para FP 40 e FP 50. Esta redução na velocidade foi realizada porque ao se aumentar a incorporação de FP o material torna-se mais rígido, e a resistência do CP até 1 minuto de ensaio deveria ser realizada segundo a exigência da normativa. O teste de impacto Izod foi efetuado utilizando um equipamento CEAST modelo Resil Impactor Junior com um pêndulo de 2,75J.

A Figura 1 apresenta um resumo da metodologia empregada para todo o desenvolvimento da pesquisa. 
Figura 1 - Metodologia utilizada para o desenvolvimento deste trabalho.

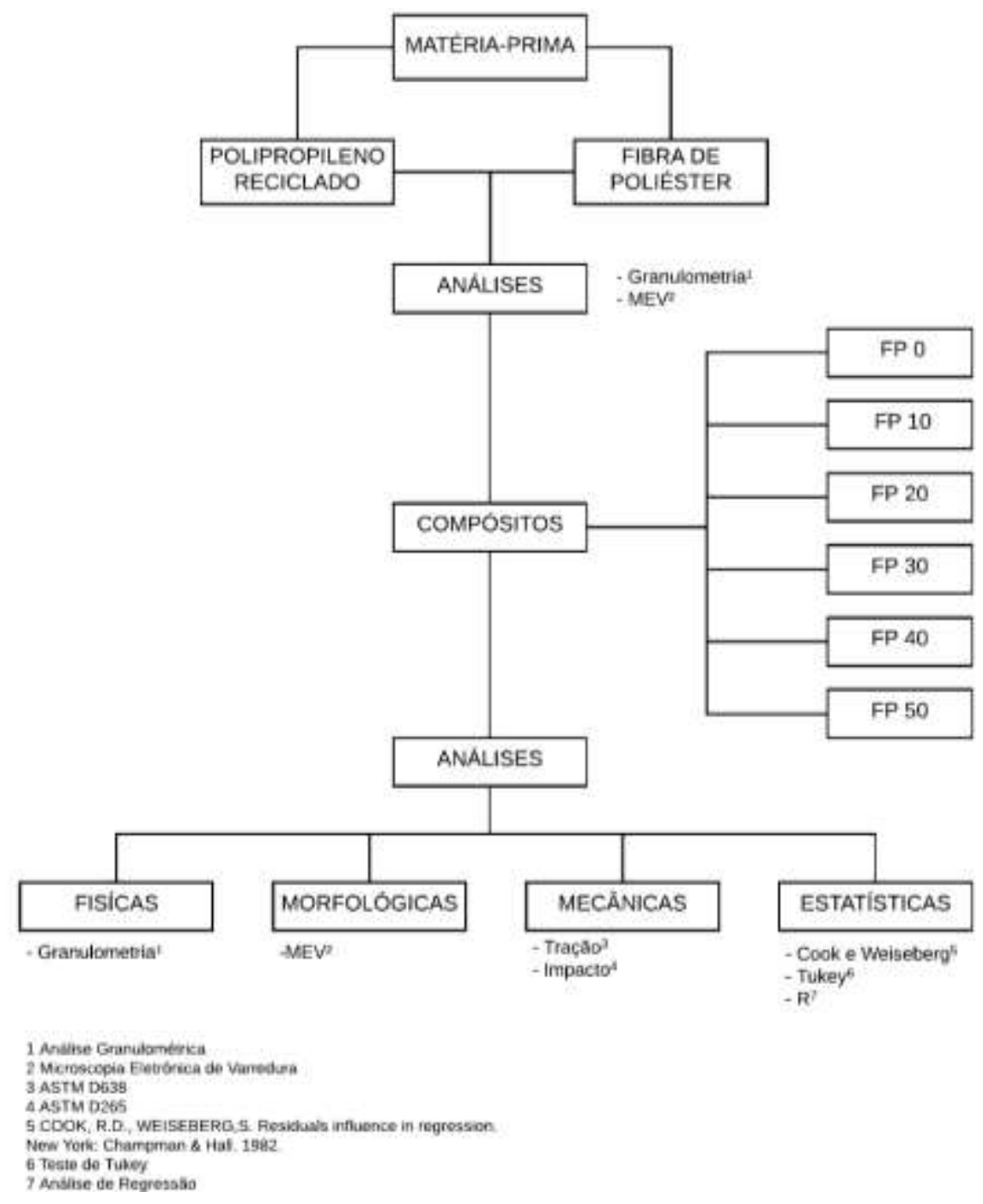

Fonte: Autores (2020).

\subsection{Análises estatísticas}

Com o intuito de verificar qual a melhor proporção da mistura de PPR e FP para ser incorporada em matriz polimérica, testou-se mecanicamente a resistência ao impacto (ASTM D256) e a resistência à tração (ASTM D638). Dos ensaios, dez e oito corpos de prova, respectivamente foram obtidos e da necessidade de se utilizar apenas cinco corpos de prova para cada análise, optou-se por realizar a análise da distância de Cook. Após a determinação dos valores dos cinco corpos de prova, aplicou-se a análise de variância com auxílio do teste de Tukey para verificar se existiam diferenças significativas entre as amostras ao nível de significância de 5\%.

Por fim, objetivando ainda verificar se as porcentagens de incorporação de FP à matriz polimérica proporcionaram uma boa resistência à tração e ao impacto, empregou-se a função desejabilidade que, com base nos valores reais determinados pelas análises mecânicas, tem como objetivo encontrar uma combinação otimizada da mistura PPR e FP. Tal método consiste em aplicar para cada variável resposta um valor adimensional que representa a sua desejabilidade individual $\left(d_{i}\right)$, expressa pela Equação 1:

$$
D=\sqrt[m]{d_{1} d_{2} d_{3} \ldots d_{m}}
$$


A otimização foi analisada levando em consideração a Equação 2:

$$
d_{i}\left(y_{i}(x)\right)=\left[\begin{array}{l}
1 \text { se } y_{i}(x)<L_{i} \\
{\left[\frac{U_{i}-y_{i}(x)}{U_{i}-L_{i}}\right]^{i}} \\
0 \text { se } y_{i}(x)>U_{i}
\end{array}\right]
$$

Eq. 2

Em relação a Equação (2) $y_{i}$ é a resposta obtida para a variável estudada, $d_{i}\left(y_{i}\right)$ é a desejabilidade individual da resposta $y_{i}, U_{i}$ é o maior valor aceitável adotado para a resposta, $L_{i}$ é o menor valor aceitável adotado para a resposta e $t$ é fator que determina o quão é desejável $y_{i}$ ser próximo do mínimo, variando de 0 a 1.

\section{Resultados e Discussão}

A análise granulométrica realizada nas matérias-primas é expressa na Tabela 2.

Tabela 2 - Análises granulométricas das fibras de poliéster (FP) e de polipropileno reciclado (PPR).

\begin{tabular}{c|c|c}
\hline Peneiras (mm) & FP (\%) & PPR (\%) \\
\hline $9,5\left(3 / 8^{\prime \prime}\right)$ & 62,91 & 0 \\
\hline $4,75\left(\mathrm{~N}^{\circ} 4\right)$ & 16,99 & 3,89 \\
\hline $2,00\left(\mathrm{~N}^{\circ} 10\right)$ & 7,66 & 83,18 \\
\hline $1,18\left(\mathrm{~N}^{\circ} 16\right)$ & 0,89 & 6,27 \\
\hline $600 \mu \mathrm{m}\left(\mathrm{N}^{\circ} 30\right)$ & 1,37 & 2,05 \\
\hline Fundo & 10,13 & 4,57 \\
\hline
\end{tabular}

Fonte: Autores (2020).

Observando os resultados obtidos pelas análises granulométricas (Tabela 2), foi possível identificar variações geométricas da FP com relação ao PPR. O estudo granulométrico mostrou que as matérias-primas apresentaram uma distribuição distinta da dimensão das partículas, pois há variação de tamanhos. Uma vez que $83,18 \%$ do PPR ficou retido em uma malha de 2,00 mm, e para a FP $62,91 \%$ correspondente a 9,5 mm e parte significante do material nas malhas com abertura de 4,75 mm, o que influencia diretamente na homogeneidade do material, sendo importante controlar a granulometria na fabricação do compósito a ser injetado (Bittencourt et al., 2018).

As análises de superfície mostram que a FP (Figura 2 (a)) é composta por estruturas independentes entre si, criando uma composição frágil. Já para a amostra de PPR (Figura 2 (b)), tem-se uma estrutura linear, com certa rugosidade, identificando assim a característica de uma matriz, pois atua com predominância devido às suas características. 
Figura 2 - Imagens da MEV das matérias-primas FP (a) e PPR (b) com a resolução de $20 \mu \mathrm{m}$.
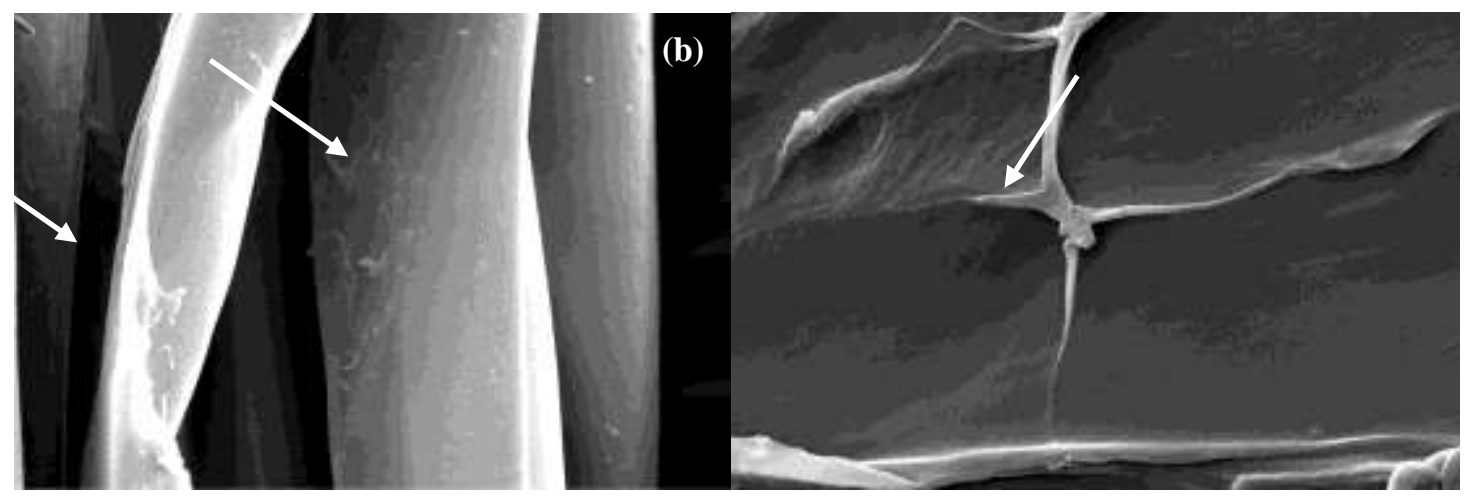

Fonte: Autores (2020).

Analisando os resultados da MEV na Figura 2 (a) percebe-se a indicação de material composto por fibras, pelo fato de possuírem espaçamentos entre si, são caracterizados como um material frágil. Já na Figura 2 (b), é verificada uma superfície homogênea e sólida. De acordo com Borsoi et al. (2011), a distribuição das fibras na matriz está diretamente relacionada ao desempenho mecânico do material, no qual a uniformidade da distribuição contribui para um melhor resultado. A identificação da característica de cada material é importante para reforçar complementariedade de ambos, ou seja, as propriedades podem ser reforçadas.

Figura 3 - Análise granulométrica amostra dos CPs.

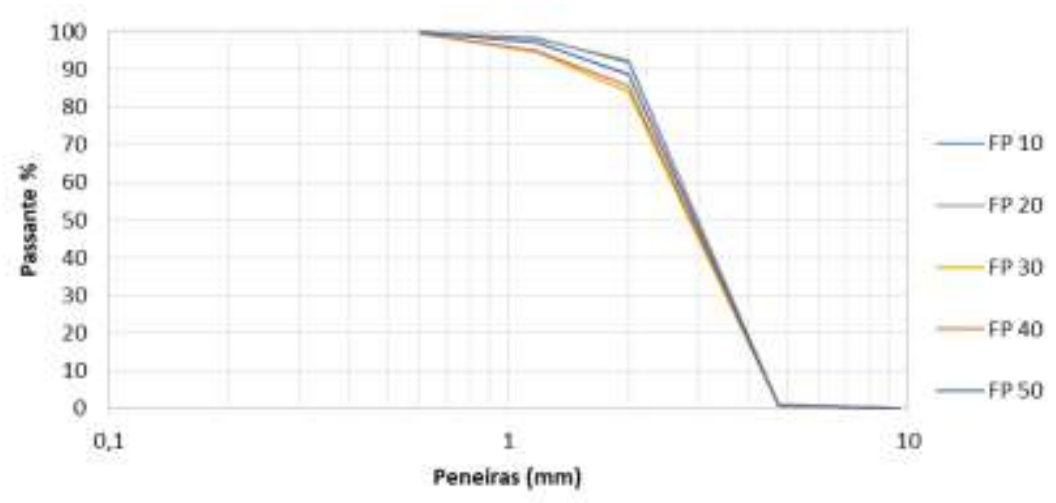

Fonte: Autores (2020).

$\mathrm{Na}$ análise granulométrica realizada para os CPs (Figura 3) foi possível verificar pouca variação granulométrica. Em média, 87,58\% das amostras ficaram retidas na peneira com abertura de 2,00 mm, aproximando-se da granulometria do PPR com maior porcentagem de material retido, seguida pela abertura de 1,18 mm. Com isso percebeu-se que a incorporação de FP nos CPs não apresentou uma diferença granulométrica significante entre eles.

Corroborando Bittencourt et al. (2018) e Firmino et al. (2017), quanto menor a granulometria, melhor a adesão à matriz, ou seja, a granulometria das matérias-primas foi ajustada para a confecção dos CPs, obtendo assim uma homogeneidade, o que tende a favorecer o desempenho mecânico.

Para verificar qual a melhor proporção da mistura de PPR e FP para ser incorporada à matriz polimérica realizou-se os ensaios de resistência a tração (ASTM D638) e ao impacto (ASTM D256). Foram ensaiados oito corpos de prova para cada incorporação de FP e no CP contendo apenas PPR, descritos na Figura 4. Após a realização dos ensaios, com o objetivo de 
eliminar os outliers que poderiam influenciar indevidamente em uma análise de regressão, foi aplicada a distância de Cook, uma vez que as normativas ASTM D638 e D256 exigem apenas 5 CPs para os ensaios (Figura 4 e 5).

Figura 4 - Análise de ruptura para todas as incorporações, pela distância de Cook (ASTM D638).
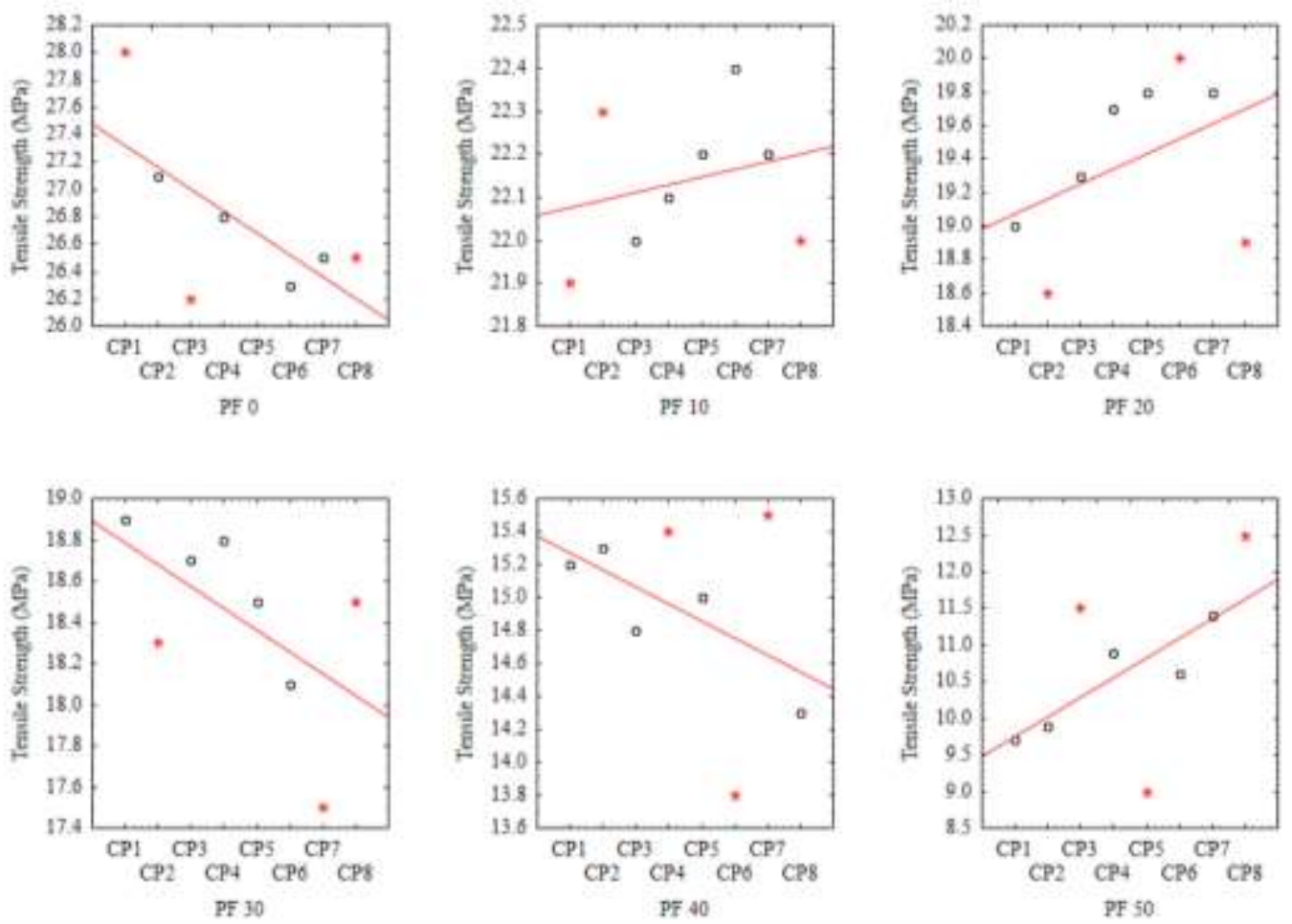

Fonte: Autores (2020).

Figura 5 - Análise de ruptura para todas as incorporações, pela distância de Cook (ASTM D256).
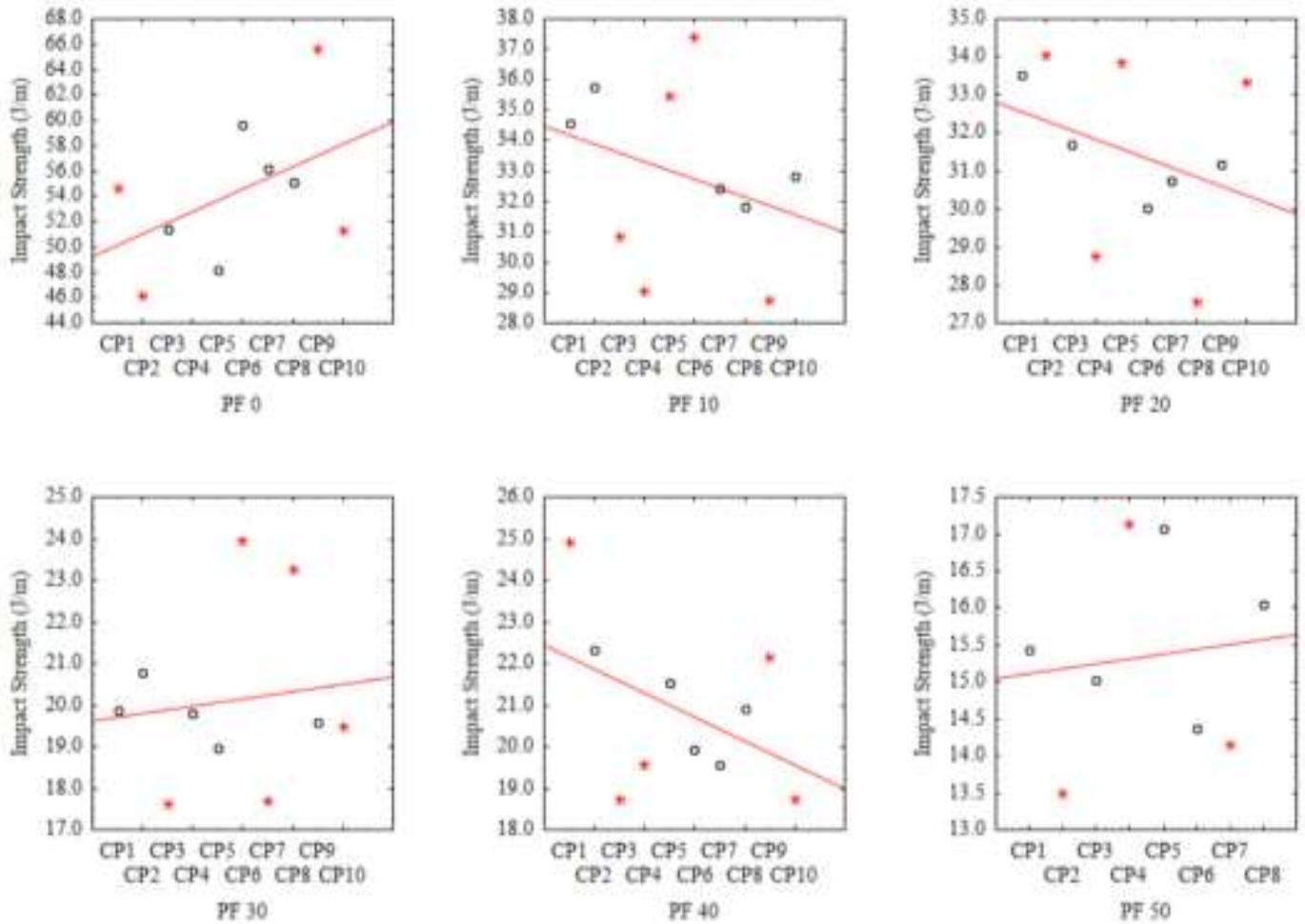

Fonte: Autores (2020). 
As Figuras 4 e 5 representam a aplicação da Distância de Cook, buscou-se assim selecionar os melhores resultados dos CPs. A metodologia pode ser compreendida da seguinte forma, a reta vermelha representa a média esperada dos resultados, então quanto mais próximo a reta melhores os resultados, os CPs eliminados estão representados em vermelho.

A eliminação dos corpos de prova pela distância de Cook foi capaz de proporcionar um melhor ajuste do $\mathrm{R}^{2}$. Desta forma, não se tem mais valores que poderiam interferir na existência de uma relação funcional entre uma variável dependente com uma ou mais variáveis independentes, além de atender às normativas expressas para o cálculo de tração e impacto segundo um número de cinco corpos de prova (Tabela 3).

Tabela 3 - Ajuste do R $^{2}$ após eliminação de corpos de prova pela distância de Cook para os ensaios de tração e impacto respectivamente.

\begin{tabular}{c|c|c|c|c|c|c}
\hline \multirow{2}{*}{$\begin{array}{c}\text { Porcentagem de } \\
\text { impregnação }\end{array}$} & \multicolumn{7}{|c}{ Variáveis obtidas } \\
\cline { 2 - 7 } & FP 0 & FP 10 & FP 20 & FP 30 & FP 40 & FP 50 \\
\cline { 2 - 7 } & \multicolumn{7}{|c}{ Tensile Strength (MPa } \\
\hline $\mathrm{R}^{2}$ & 0,4568 & 0,0674 & 0,1831 & 0,3300 & 0,1840 & 0,2262 \\
\hline $\mathrm{R}^{2}$ após eliminação dos CPs & 0,7769 & 0,5568 & 0,8681 & 0.8100 & 0,7783 & 0,8490 \\
\hline & \multicolumn{7}{|c|}{ Impact Strength $\left(\mathrm{Jm}^{-1}\right)$} \\
\hline $\mathrm{R}^{2}$ & 0,2028 & 0,1088 & 0,3881 & 0,0574 & 0,0544 & 0,0319 \\
\hline $\mathrm{R}^{2}$ após eliminação dos CPs & 0,2538 & 0,5393 & 0,4635 & 0,3413 & 0,4443 & 0,2126 \\
\hline
\end{tabular}

Fonte: Autores (2020).

Após a determinação dos CPs aplicou-se a análise de variância com auxílio do teste de Tukey para verificar se existiam diferenças significativas entre as amostras ao nível de significância de 5\%. Os resultados das médias e dos desvios padrões para a resistência em relação ao impacto nas seis composições testadas encontram-se na Tabela 4.

Tabela 4 - Médias e desvios padrões dos testes realizados com a incorporação de FP.

\begin{tabular}{ccc}
\hline Amostras & $\begin{array}{c}\text { Resistência à Tração } \\
(\mathbf{M P a})\end{array}$ & $\begin{array}{c}\text { Resistência ao } \\
\text { Impacto } \\
(\mathbf{J} / \mathbf{m})\end{array}$ \\
\hline FP 0 & $26,66^{\mathrm{a}} \pm 0,14$ & $53,92^{\mathrm{a}} \pm 2,43$ \\
FP 10 & $22,18^{\mathrm{b}} \pm 0,07$ & $33,42^{\mathrm{b}} \pm 0,68$ \\
FP 20 & $19,52^{\mathrm{c}} \pm 0,16$ & $31,50^{\mathrm{b}} \pm 0,65$ \\
FP 30 & $18,60^{\mathrm{b}} \pm 0,14$ & $19,81^{\mathrm{cd}} \pm 0,30$ \\
FP 40 & $15,14^{\mathrm{e}} \pm 0,11$ & $20,86^{\mathrm{c}} \pm 0,51$ \\
FP 50 & $10,50^{\mathrm{f}} \pm 0,31$ & $15,60^{\mathrm{d}} \pm 0,46$ \\
\hline
\end{tabular}

*Sobrescritos iguais na mesma coluna, para a mesma variável resposta, indicam médias sem diferença estatisticamente significativa com 5\% no teste de Tukey. Fonte: Autores (2020).

Segundo os dados apresentados na Tabela 4, é possível verificar que, à medida que se incorpora a FP, há uma redução da resistência ao impacto. No entanto, não há uma diferença significativa entre as amostras com FP 10 e FP 20. Também não há entre FP 30 e FP 40. E esta, porém, não se difere da amostra com FP 50. Com relação à resistência à tração, também há uma diminuição à medida que se incorpora a FP na matriz polimérica e, pelo teste de Tukey, não houve semelhança significativa entre as médias (Beh et al., 2018).

A análise de superfície de resposta (Figura 6) apresenta a melhor combinação entre as variáveis analisadas, confirmando os resultados obtidos do teste de Tukey, ou seja, à medida que se incorpora a fibra de poliéster, há uma diminuição sutil na resistência à tração e a resistência ao impacto. Resultados também apresentados por Spadetti et al., (2017), ao incorporar $40 \%$ de fibra de celulose em matriz polimérica obteve uma redução de resistência, e isso se deve a aglomeração 
das fibras, sendo justificada pela baixa adesão entre fibra e matriz. De modo que o compósito apresenta maior rigidez que o PPR puro, tornando-se assim um compósito fragilizado.

De acordo com as análises de superfície (MEV) obtidas para a matéria-prima PPR e FP, foi possível identificar superfícies distintas, podendo dessa forma justificar a redução da resistência dos compósitos quando incorporados (Barbosa, 2019; Borsoi et al., 2011).

Figura 6 - Superfície de resposta com a incorporação da fibra de poliéster.

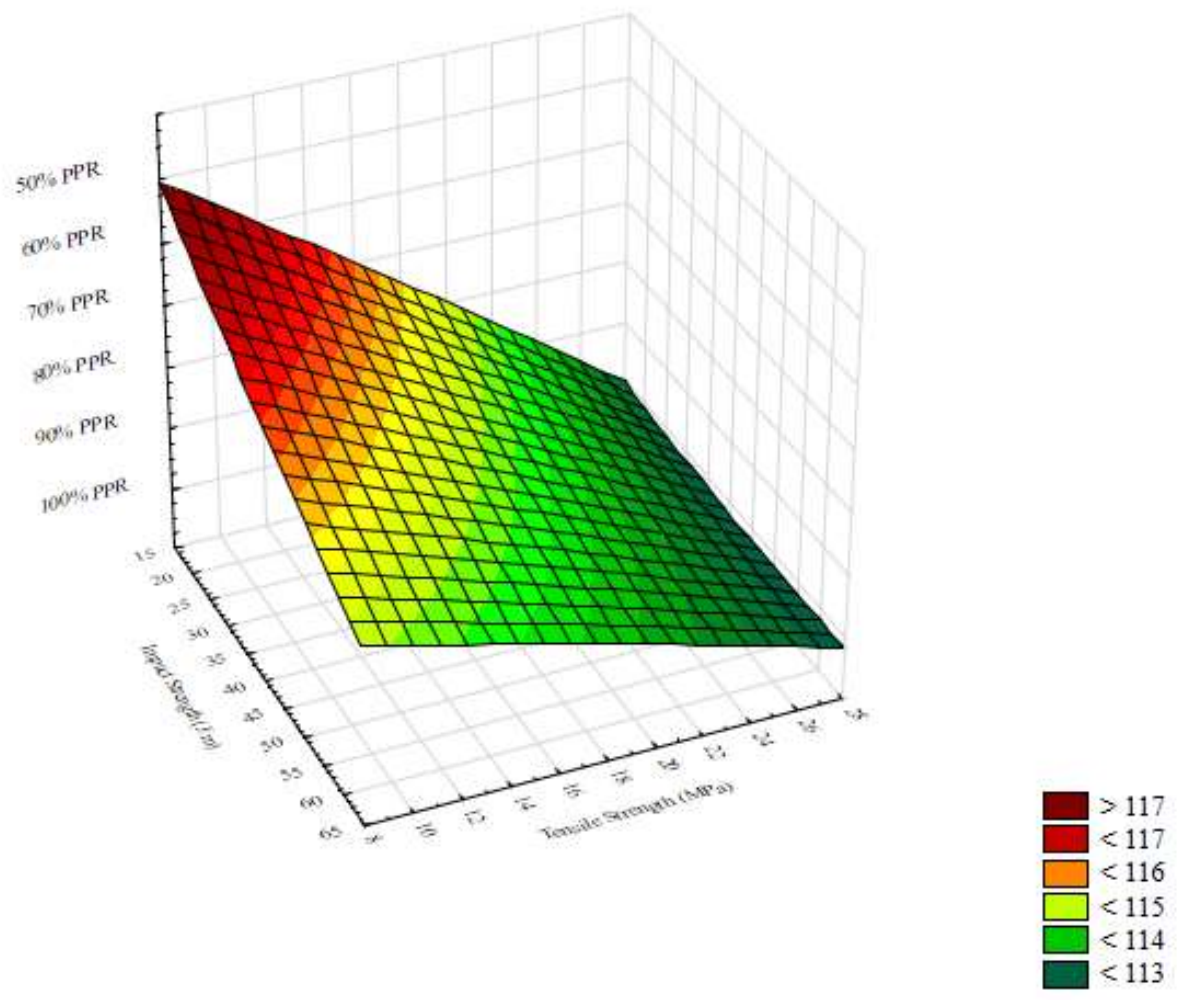

Fonte: Autores (2020).

A Análise de superfície representada na Figura 6 pode ser compreendida da seguinte forma, quanto maior a incorporação de fibra menor a resistência do material, observamos esse fato ao analisar o eixo com os valores das incorporações. Este é um comportamento esperado, pelo fato de que quanto maior a incorporação de fibra, mais rígido fica o material.

Robki et al. (2019) também verificaram a variação em relação à resistência à tração em suas amostras, utilizando o polipropileno reciclado com a incorporação de juta simples. Utilizando a variante de temperatura e pressão, os resultados apresentaram valores entre 14 e $24 \mathrm{MPa}$. Outra pesquisa utilizando compósito laminados de polipropileno reforçado com juta/linho (Singh et al., 2019) teve uma variação de resistência a tração de 17,82 a 20,36 MPa.

De acordo com Suharty et al., (2016), o polipropileno reciclado possui uma tensão de ruptura 21,25 MPa e, com a incorporação de fibra kenaf (FK), houve um aumento da resistência à tração de $18 \%$ e resistência ao impacto de $27 \%$. Os

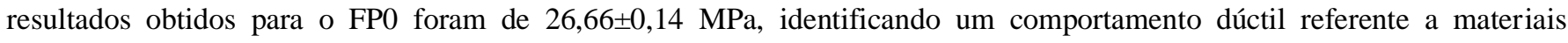
termoplásticos, elevada deformação na ruptura com resistência à tração e módulos baixos. Sendo que seus resultados são referência para as demais incorporações (Becker et al., 2011; Silva et al., 2019). 
Já nos demais CPs com a adição de FP, foi possível identificar um comportamento frágil, conforme ocorria o aumento de FP, com deformação linear em baixas tensões e uma redução significativa no estiramento e elevado aumento no módulo de elasticidade, comportamento característico de materiais compósitos (Callister, 2012).

Figura 7 - Análise dos resultados de tração (ASTM D638), em relação à ductilidade (\%) e o módulo de elasticidade (MPa).

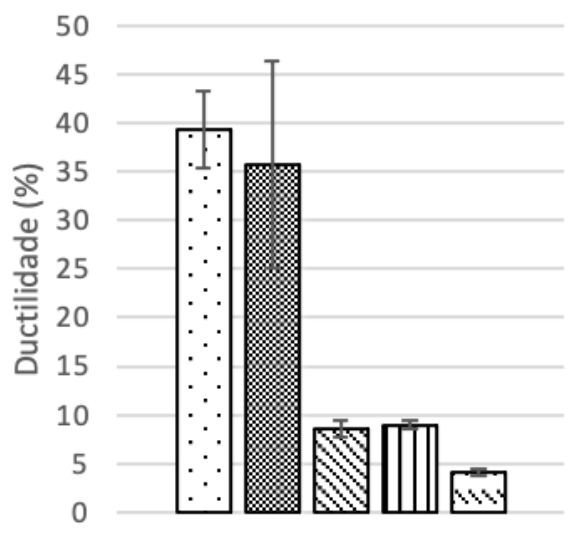

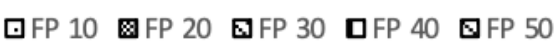

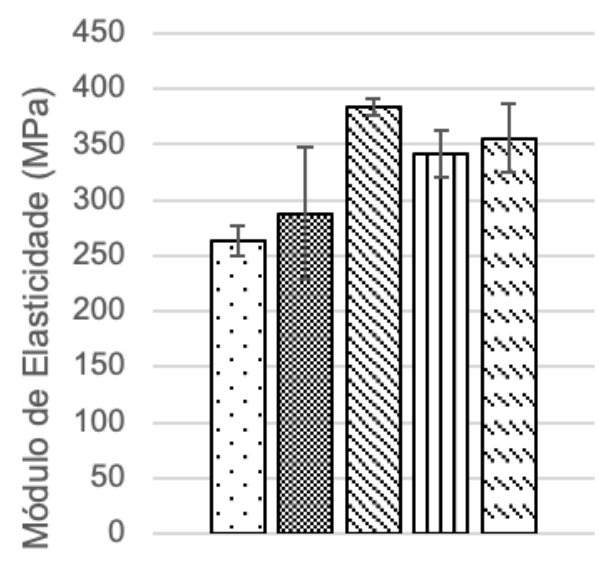

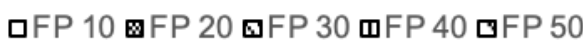

Fonte: Autores (2020).

De acordo com a Figura 7, o mesmo ocorreu em relação ao parâmetro de ductilidade (\%), referindo-se ao alongamento, ou seja, o grau de deformação plástica que foi suportado até a fratura, teve uma redução de 89,53\% dos CPs entre FP 10 e FP50. O aumento da incorporação de FP influencia diretamente na redução do comportamento dúctil da matriz polimérica. Possível de ser identificado o comportamento dúctil nas amostras FP0, FP10 e FP20 e comportamento frágil em FP30, FP40 e FP50. O mesmo aconteceu para Arsad et al., (2013), que trabalhou com a influência da fibra kenaf como reforço nos compósitos de PPR, a resistência à tração sem kenaf foi de $31 \mathrm{MPa}$ e quando incorporada $10 \%$ de fibra este valor reduziu para $27 \mathrm{MPa}$, justificada pela baixa dispersão da fibra na matriz polimérica.

Em relação aos resultados mecânicos, é preciso avaliar o tipo de fibra que foi incorporada na matriz polimérica e a forma que o processo foi realizado. Todos estes fatores influenciam na forma que o material se comporta mecanicamente (Todkar et al., 2019). De acordo Borsoi et al., (2011), as matrizes poliméricas quando reforçadas com fibras têxteis, possuem uma carência de compatibilidade adequada entre fibra e matriz, fazendo com que a adesão interfacial não ocorra de forma adequada, influenciando diretamente nos resultados mecânicos. A utilização de um agente compatibilizante é necessária para que se tenha um melhor desempenho das propriedades mecânicas. Como relatado por Gupta et al. (2014), que utilizou diversos elementos químicos como silano, metacrilato de glicidil (GMA) e cloreto de O-hidroxibenzeno diazônio (OBDC) para melhorar a compatibilidade entre a superfície da fibra e a matriz polimérica. Os resultados foram positivos, os ensaios de tração tiveram um aumento de $11 \%, 20 \%$ e $31,36 \%$ e resistência ao impacto de 78,72\%, $77 \%$ e $81 \%$ para os compósitos de polipropileno reciclado reforçado com fibra de sisal tratados com silano, GMA e OBDC, respectivamente, quando comparados ao PPR.

Ainda de acordo com o autor supracitado, para a incorporação de resíduos de fibra de algodão em uma matriz de polietileno foram feitos comparativos entre CPs com e sem agente compatibilizante, os resultados mecânicos foram superiores ao CP de referência (PS). O módulo de elasticidade para a incorporação de $20 \%$ de fibra de algodão com compatibilizante obteve o melhor resultado para o ensaio de tração, aproximadamente $50 \mathrm{MPa}$. Já para o ensaio de impacto, o melhor resultado 
para os compósitos com $20 \%$ em massa de fibra de algodão, sem agente compatibilizante, foi de 253,05 J.m ${ }^{-1}$ em comparação aos $135,59 \mathrm{~J} . \mathrm{m}^{-1}$ obtidos para a matriz de PS, o que corresponde a um desempenho $86 \%$ superior ao da matriz.

Em outras pesquisas, utilizando compósitos de polipropileno reciclado com a incorporação de fibra Kenaf e adição de agentes compatibilizantes, os resultados para a resistência à tração variaram de 32,32 a 38,30 MPa (Suharty et al., 2016).

Os resultados, considerando a incorporação de fibra, encontrados no presente trabalho foram inferiores ao CP de referência (FP0), isso pode ser justificado pelo fato de que a FP utilizada para ser o material de reforço é reciclada e pode ter perdido parte de suas propriedades nesse processo, pois o módulo de elasticidade do PPR apresenta um valor de 326,69 MPa e quando incorporado 10\% de fibra, esse valor reduz para 263,61 MPa, uma redução de 19,31\%. Já para a incorporação de FP30, houve um aumento de $17 \%$, isso pode ser justificado pela melhor aderência entre fibra e matriz, como pode ser observado na análise de superfície disposta na Figura $8(\mathrm{~d})$ e $\left(\mathrm{d}_{1}\right)$. Os valores obtidos para resistência à tração, do PPR apresenta um valor.

As imagens obtidas por microscopia eletrônica de varredura (MEV) foram realizadas nos corpos de prova rompidos no ensaio de impacto e estão dispostas na Figura 7, para todas as incorporações, de FP0 à FP50.

As figuras representam o material após a fratura, para a amostra FP0 (a) e (a $a_{1}$ ), é possível observar uma homogeneidade do material por se tratar do PPR sem incorporação de fibra. Nas imagens de $b$ à $f_{1}$, se observa a presença da fibra e os vazios existentes entre ela e a matriz, sendo essa uma característica mais evidente nas incorporações FP 10, FP 20, FP 40 e FP 50. A interação fragilizada entre a matriz e a fibra poderá prejudicar as propriedades mecânicas dos compósitos mas, com a utilização de um agente compatibilizante (Borsoi et al., 2011; Suharty et al., 2016), ocorre uma interação entre as fibras e o polipropileno e os espaços vazios entre estes materiais são eliminados, proporcionando uma melhoria das propriedades mecânicas.

Figura 8 - Imagens da MEV da fratura dos CPs, referente a cada incorporação: (a) e (a $\left.a_{1}\right)$ FP0; (b) e (b 1 ) FP10; (c) e (c 1 ) FP20;

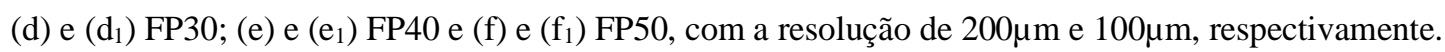
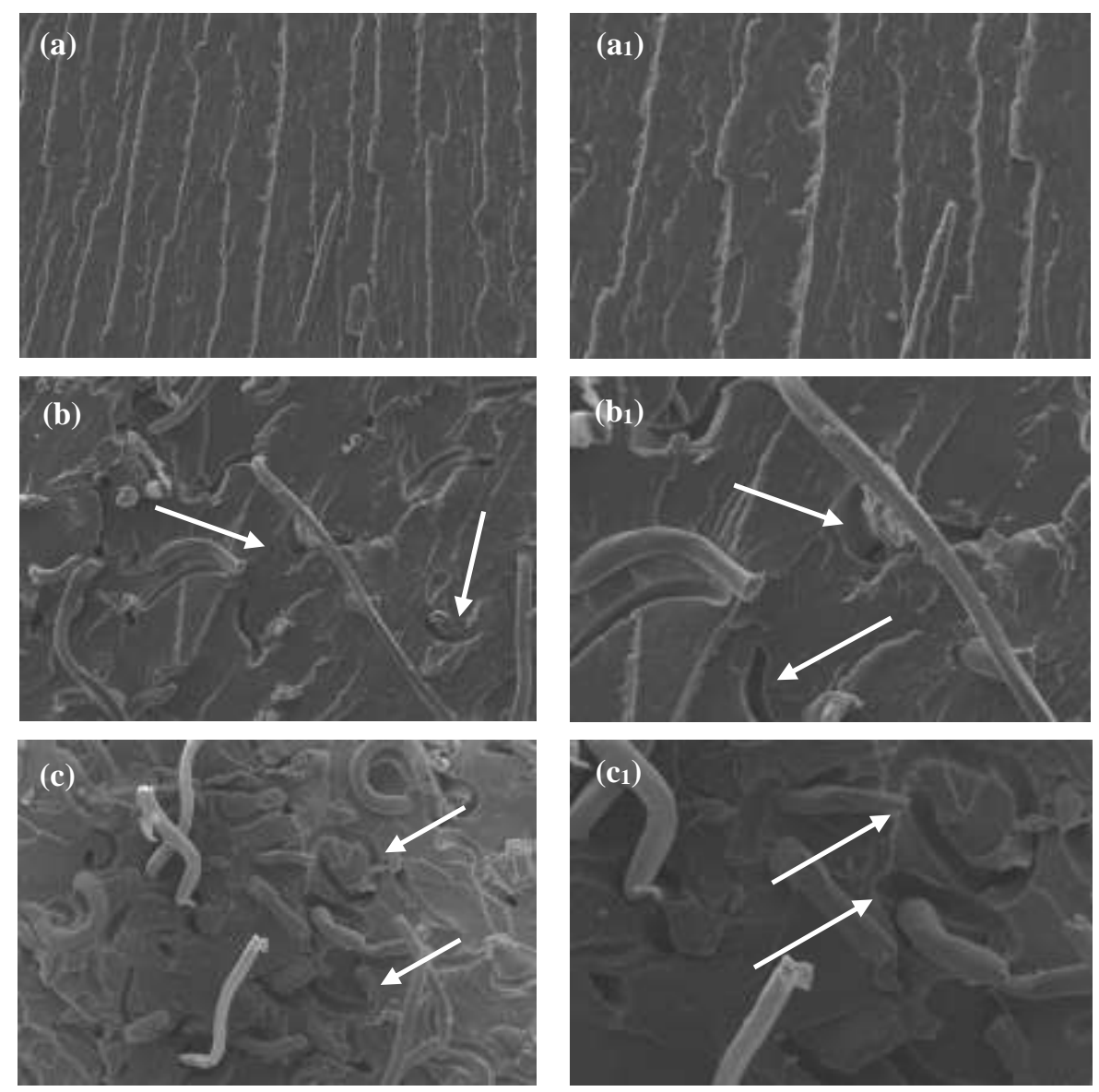
Research, Society and Development, v. 10, n. 2, e27910212215, 2021

(CC BY 4.0) | ISSN 2525-3409 | DOI: http://dx.doi.org/10.33448/rsd-v10i2.12215
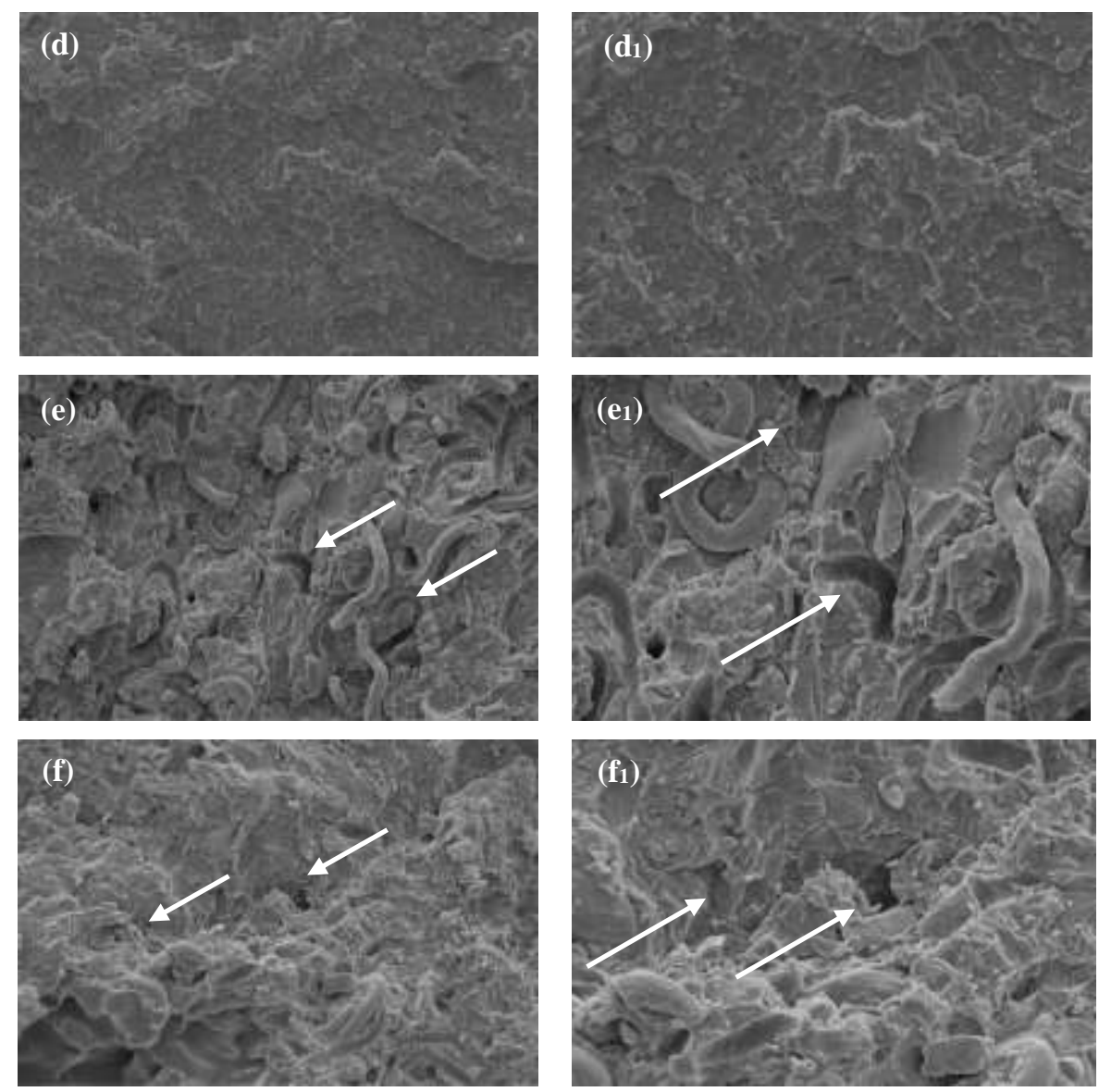

Fonte: Autores (2020).

E considerando o aspecto ambiental, de forma a reduzir a necessidade de recursos, optou-se pela não utilização de compatibilizantes, uma vez que a utilização do menor número de matéria-prima colabora para sustentabilidade do meio ambiente, além de minimizar o impacto ambiental e colaborar para a preservação, haja vista toda a matéria-prima ser reciclada. 
Figura 9 - Gráfico de influência da mistura de PPR e FP na resistência ao impacto e a resistência à tração. PPR
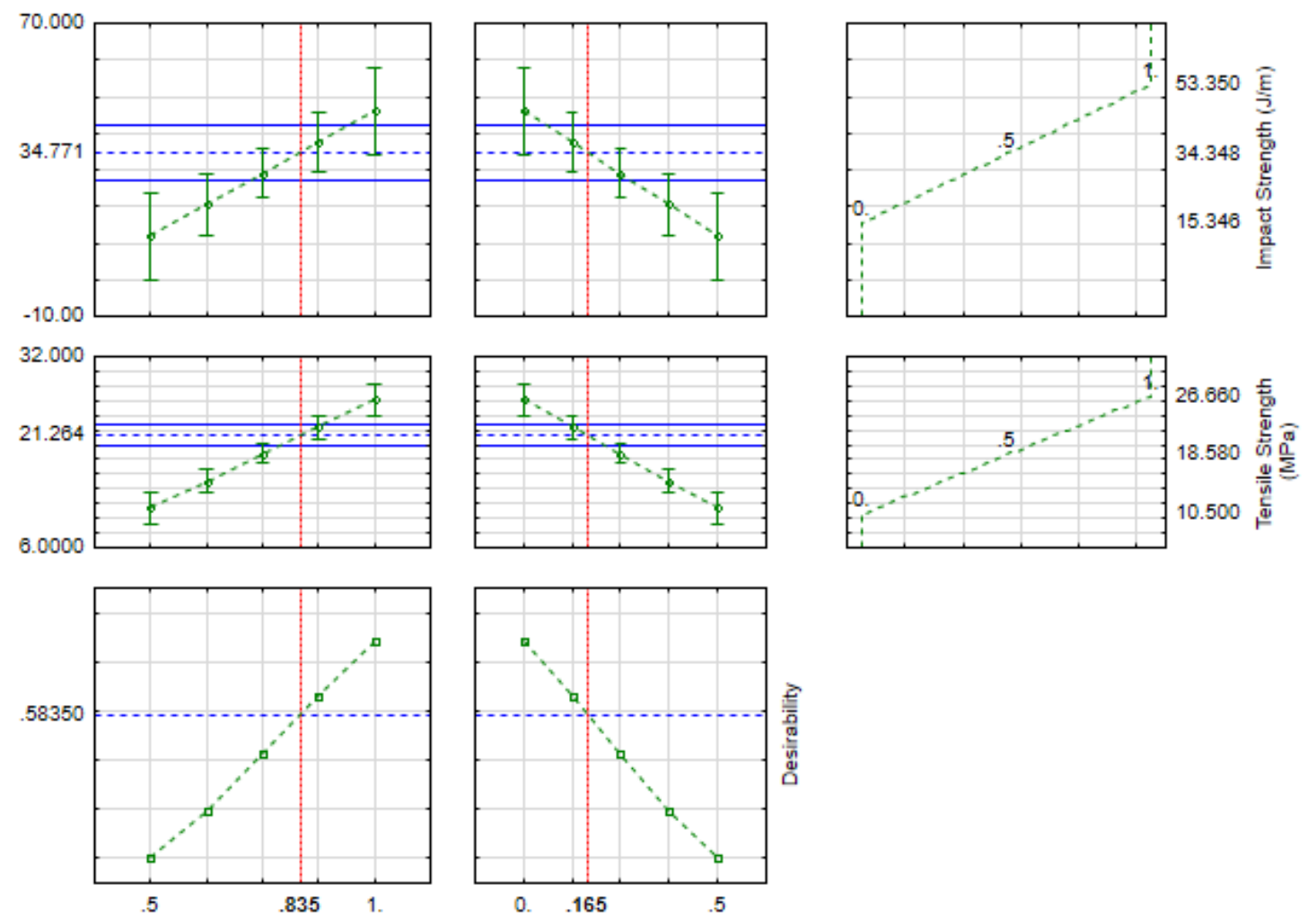

Fonte: Autores (2020).

Com a finalidade ainda de verificar se as porcentagens de impregnação de fibra de poliéster à matriz polimérica proporcionaram uma boa resistência à tração e ao impacto, empregou-se a função desejabilidade que, com base nos valores reais determinados pelas análises mecânicas, tem como objetivo encontrar uma mistura otimizada de PPR e FP. Na Figura 9 é possível verificar esta análise que, numericamente, seria interessante colocar 83,5\% de PPR e 16,5\% de FP (Gomes et al., 2019; Triñanes et al., 2019).

Com esta proporção tem-se uma aproximação de 58,35\% em relação aos dados experimentais encontrados, ou seja, se a ideia de impregnar a FP na matriz polimérica era um bom desempenho mecânico, esta é a mistura otimizada. A partir dessa formulação, foram inferidos ensaios de resistência à tração e ao impacto. Da mesma forma que os ensaios anteriores, oito corpos de prova foram testados para a resistência à tração e dez corpos para a resistência ao impacto. A eliminação dos corpos que excediam à norma vigente também aconteceu por meio da distância de Cook, apresentadas na Figura 10 e Tabela 5, respectivamente, bem como seus respectivos ajustes $\left(\mathrm{R}^{2}\right)$ antes e após a eliminação, respectivamente. 
Figura 10 - Análise de ruptura para a formulação otimizada, pela distância de Cook.
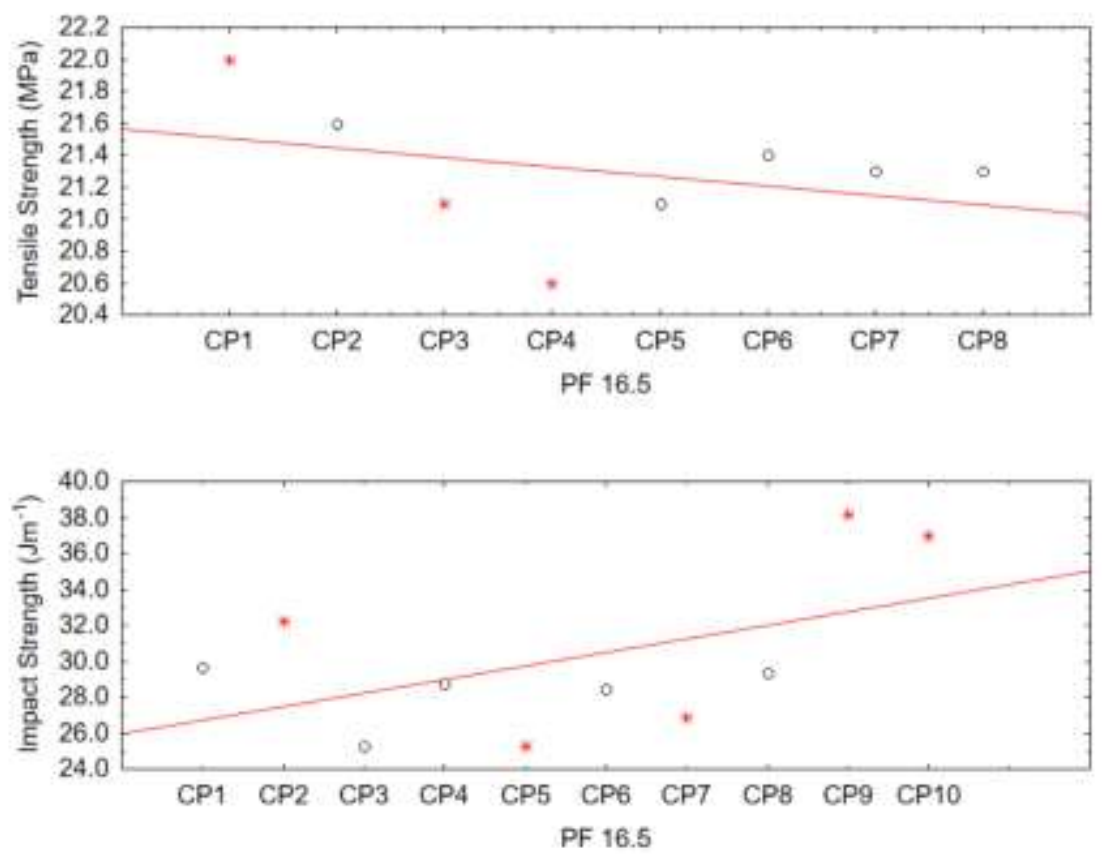

Fonte: Autores (2020).

A Figura 10 representa a aplicação da Distância de Cook, a mesma explicada anteriormente (Figuras 4 e 5), tendo o intuito de selecionar os melhores CPs em relação as análises de impacto e tração.

Tabela 5 - Corpos de prova e distância de Cook.

\begin{tabular}{c|c|c}
\hline \multirow{2}{*}{ CPs } & \multicolumn{2}{|c}{ Distância de Cook } \\
\cline { 2 - 3 } & $\begin{array}{c}\text { ASTM D256 } \\
\text { Impacto }\left(\mathrm{Jm}^{-1}\right)\end{array}$ & $\begin{array}{c}\text { ASTM D638 } \\
\text { Tração (MPa) }\end{array}$ \\
\cline { 2 - 3 } & FP 16.5 & FP 16.5 \\
\hline CP1 & -- & -- \\
\hline CP2 & -- & 0,035212 \\
\hline CP3 & 0,067299 & -- \\
\hline CP4 & 0,000240 & -- \\
\hline CP5 & 0,076766 & 0,014908 \\
\hline CP 6 & 0,016322 & 0,028132 \\
\hline CP 7 & -- & 0,034111 \\
\hline CP 8 & 0,053899 & 0,157672 \\
\hline CP 9 & -- & -- \\
\hline CP 10 & -- & -- \\
\hline R & 0,2647 & 0,1283 \\
\hline $\mathrm{R}^{2}$ após eliminação & 0,4241 & 0,3025 \\
\hline Média & $27,43 \pm 1,99$ & $21,34 \pm 0,18$ \\
\hline
\end{tabular}

Fonte: Autores (2020).

Na prática foi possível verificar que a tração para a formulação otimizada proporcionou o mesmo valor $(21,34 \pm 0,18$ $\mathrm{MPa}$ ) proposto pela função desejabilidade (21,26 MPa). Com relação ao impacto, o valor encontrado $\left(27,43 \pm 1,99 \mathrm{Jm}^{-1}\right)$ ficou cerca de $15 \%$ menor do que o valor proposto teoricamente. Desta forma é possível destacar a importância de se realizar cálculos para valores teóricos à priori no intuito de minimizar tempo e custo para o pesquisador e para profissionais das mais diferentes áreas, contribuindo com a preservação ambiental. 


\section{Conclusão}

A utilização de um material compósito que possui matérias-primas que seriam consideradas resíduos pode contribuir para a preservação ambiental, pois no presente trabalhou buscou-se avaliar as propriedades mecânicas da incorporação de fibra de poliéster em matriz de polipropileno reciclado. Embora os resultados não tenham superado o material puro, contribuíram para a redução de resíduos e dentre as incorporações apresentadas, foi encontrada a composição ideal de incorporação.

Com uma formulação otimizada que considerou uma incorporação de $16,5 \%$ de fibra de poliéster, os resultados obtidos para o ensaio de resistência à tração (21,34 Mpa) e para resistência ao impacto (27,43 Mpa) foram significativos, ainda mais considerando o aspecto de resistência mecânica do material e, principalmente, a função ecológica de minimizar resíduos no meio ambiente.

Assim, para estudos futuros tendo como objetivo o aprimoramento do material, considerar ensaios de outros parâmetros e até mesmo desenvolvimento de outras técnicas de incorporação das fibras de poliéster, com o intuito de melhorar as propriedades do material desenvolvido.

\section{Agradecimentos}

Agradecimento a Capes pela concessão da bolsa de estudos e ao laboratório de fabricação mecânica da UEM pelo auxílio nas análises mecânicas.

\section{Referências}

Aciu, C., Ilutiu-varvara, D-A, Manea, D-L., Orban, Y-A., \& Babora, F. (2018). Recycling of plastic waste materials in the composition of ecological mortars. Procedia Manufacturing, 22, 274-279.

Albach, B., Vianna, S. P. H, Rampon, D. S., \& Barbosa, R.V. (2019) An evaluation of modified Kaolinite surface on the crystalline and mechanical behavior of polypropylene. Polymer Testing, 75, 237-245.

Ali Mohsin, M. E., Ibrahim, A. N., Arsad, A., ABD Rahman, M. F., \& Alothman, O. Y. (2015) Effect of polypropylene, ethylene vinyl acetate and polyamide6 on properties of recycled polypropylene/empty fruit bunch composites. Fibers and Polymers, 16, 2359-2367.

Amorim, C. C., Leão, M. M. D., \& Moreira, R. F. P. M. (2019). Comparação entre diferentes processos oxidativos avançados para degradação de corante azo. Engenharia Sanitária e Ambiental, 14, 543-550.

Arsad, A., Suradi, N. L., Rahmat, A. R., Danlami, J. M. (2013). The influence of kenaf fiber as reinforcement on recycled polypropylene/recycled polyamide-6 composites. International Journal of Plastics Technology, 17, 149-162.

American Society For Testing And Materials. ASTM D256-10e1: standard test methods for determining the izod pendulum impact resistance of plastics. Philadelphia, 2010.

American Society For Testing And Materials. ASTM D638-10: standard test method for tensile properties of plastics. Philadelphia, 2010.

Aygormez, Y., Canpolat, O., AL-MashhadanI, M. M., \& Uysal, M. (2020). Elevated temperature, freezing-thawing and wetting-drying effects on polypropylene fiber reinforced metakaolin based geopolymer composites. Construction and Building Materials, 235, $117-502$.

Barbosa, J. T. L. Caracterização de compósitos poliméricos feitos com resíduos pós-consumo (poliestireno de alto impacto) e industriais (lama vermelha e fibra de vidro/resina epóxi). Dissertação, Universidade Estadual Paulista Júlio Mesquita Filho, São Paulo, 2019.

Becker, D., Kleinschmidt, A. C., Balzer, P. S., \& Soldi, V. (2011). Influência da sequência de mistura do PP-MA nas propriedades dos compósitos de PP e fibra de bananeira. Polímeros, 21, 7-12.

Bedekar, P. A., Bhalkar, B. N., Patil, S. M., \& Govindwar, S. P. (2016). Moringa oleifera-mediated coagulation of textile wastewater and its biodegradation using novel consortium-BBA grown on agricultural waste substratum. Environmental Science and Pollution Research, 23, $20963-20976$.

Beh, E. J., Lombardo, R., \& Alberti, G. (2018). Correspondence analysis and the Freeman-Tukey statistic: A study of archaeological data. Computational Statistics \& Data Analysis, 128, 73-86. 2018.

Bittencourt, A., Mariot, H., Ribeiro, A., Campos, D., Marques, C., \& Resmini, M. A. (2018). Estudo da Influência da Granulometria e da Carga Mineral em Resina Epóxi Utilizada em Fachada Ventilada. Cerâmica industrial, .23, 13-23.

Borsoi, C., Scienza, L. C., Zattera, A. J., \& Angrizani, C. C. (2011). Obtenção e caracterização de compósitos utilizando poliestireno como matriz e resíduos de fibras de algodão da indústria têxtil como reforço. Polímeros, 21, 271-279. 
Carpio, M., Roldán-Fontana, J., Pacheco-Torres, R., \& García, J. (2016). Construction waste estimation depending on urban planning options in the design stage of residential buildings. Construction and Building Materials, 113, 561-570.

Costa, M. A. (2018). Como avaliar o alcance dos Objetivos de Desenvolvimento Sustentável? Desafios e possibilidades para a agenda global de avaliação. Revista Brasileira de Políticas Pública e Internacionais, 3, 100-123.

Dissanayake, D. G. K., Weerasinghe, D. U., Wijesinghe, K. A. P., \& Kalpage, K. M. D. M. P. (2018). Developing a compression moulded thermal insulation panel using postindustrial textile waste. Waste Management, 79, 356-361.

Djonú, P., Rabelo, L. S., Lima, P. V. P. S., Souto, M. V. S., Sabadia, J. A. B., \& Sucupira Junior, P. R. G. (2018). Objectives of sustainable development and conditions of health risk areas. Ambiente \& Sociedade, 21.

Dria, R., Gasperi, J., Rocher, V., \& Tassin, B. Synthetic and non-synthetic anthropogenic fibers in a river under the impact of Paris Megacity: Sampling methodological aspects and flux estimations. Science of The Total Environment, 618, 157-164. 2018. https://doi.org/10.1016/j.scitotenv.2017.11.009

Echeverria, C. A., Pahlevani, F., Handoko, W., Jiang, C., Doolan, C., \& Sahajwalla, V. (2019). Engineered hybrid fibre reinforced composites for sound absorption building applications. Resources, Conservation and Recycling, 43, 1-14.

Firmino, H. C. T., Chagas, T. F., Melo, P. M. A., \& Silva, L. B. (2017). Caracterização de compósitos particulados de polietileno de alta densidade/pó de concha de molusco. Matéria, 22.

Freeman, M. F., \& Tukey, J. W. (1950). Transformations Related to the Angular and the Square Root Ann Math Statist. 21, 607-611.

Gupta, A., Biswal, M., Mohanty, S., \& Nayak, S. (2014). Mechanical and Thermal Degradation Behavior of Sisal Fiber (SF) Reinforced Recycled Polypropylene (RPP). Composites Fibers and Polymers, 15, 994-1003.

Haslinger, S., Hummel, M., Anghelescu-Hakala, A., \& Maettanem, S. H. (2019). Upcycling of cotton polyester blended textile waste to new man-made cellulose fibers. Waste Management, 97, 88-96.

Hu, Y., Du, C., Leu, S.Y., Jing, H., Li, X., \& Lin, C. S. K. (2018). Valorisation of textile waste by fungal solid state fermentation: An example of circular waste-based biorefinery. Resources, Conservation and Recycling, 129, 27-35.

Karbalaei, S., Hanachi, P., Walker, T. R., \& Cole, M. (2018). Occurrence, sources, human health impacts and mitigation of microplastic pollution. Environmental Science and Pollution Research, 25, 36046-36063.

Klapiszewski, L. et al. (2019). Kraft lignin/cubic boron nitride hybrid materials as functional components for abrasive tools. International Journal of Biological Macromolecules, 122, 88-94.

Martin, L., \& Perry, F. Sustainable Construction Technology Adoption. In: Tam VWY, Le KN. (2019). Sustainable construction technologies, $299-316$.

Militky, J. (2018). Tensile failure of polyester fibers. In: Bunsell AR (ed) Handbook of Properties of Textile and Technical Fibres (Second Edition). Woodhead Publishing, 421-514.

Nielfa, A., Cano, R., \& FDZ-Polanco, M. (2015). Theoretical methane production generated by the co-digestion of organic fraction municipal solid waste and biological sludge. Biotechnology reports, 5, 14-21.

Novaes, C. G., Yamaki, R. T., \& Paula, V. F., Nascimento Júnior, B. B., Barreto, J. A., Valasques, G. S., Bezerra, M. A. (2017). Otimização de métodos analíticos usando metodologia de superfícies de resposta. Revista Virtual de Química.

Obaid, N., Kortschot, M. T., \& Sain, M. (2018).Predicting the stress relaxation behavior of glass-fiber reinforced polypropylene composites. Composites Science and Technology, 161, 85-91.

Ogunola, O. S., Onada, O. A., \& Falaye, A. E. (2018). Mitigation measures to avert the impacts of plastics and microplastics in the marine environment (a review). Environmental Science and Pollution Research, 25, 9293-9310.

Pereira A. S. et al. (2018). Metodologia da pesquisa científica. UFSM.

Qin, Y., Zhang, X., Chai, J., Xu, Z., \& Li, S. (2019). Experimental study of compressive behavior of polypropylene-fiber-reinforced and polypropylene-fiberfabric-reinforced concrete. Construction and Building Materials, 194, 216-225.

R D Cook, \& S Weisberg (1982) Residuals and influence in regression. Chapman and Hall, London

Rokbi, M., Khaldoune, A., Sanjay, M. R., Senthamaraikannan, P., Ati, A., \& Siengchin, S. (2019). Effect of processing parameters on tensile properties of recycled polypropylene based composites reinforced with jute fabrics. International Journal of Lightweight Materials and Manufacture, 3, $144-149$.

Saccani, A., Manzi, S., Lancellotti, I., \& Lipparini, L. (2019). Composites obtained by recycling carbon fibre/epoxy composite wastes in building materials. Construction and Building Materials, 04, 296-302.

Silva, D. N. S. (2017). Estudo e Caracterização Mecânica de Compósitos de Matriz Polimérica Reforçado com Fibras de Eucalipto. Dissertação, Universidade do Porto.

Silva, G. C., Ladeira, G. F. X., Nascimento Júnior, H., \& Carneiro, J. R. G. (2019). Estudo da substituição do nylon por compósito de polipropileno com fibra de vidro. Matéria.

Sharma, S., Sudhakara, P., Nijar, S., Saini, S., \& Singh, G. (2018). Recent Progress of Composite Materials in various. Novel Engineering Applications Materials Today: Proceedings, 5, 28195-28202. 
Research, Society and Development, v. 10, n. 2, e27910212215, 2021 (CC BY 4.0) | ISSN 2525-3409 | DOI: http://dx.doi.org/10.33448/rsd-v10i2.12215

Spadetti, C., Silva Filho, E. A., Sena, G. L., \& Melo, C. V. P. (2017). Propriedades térmicas e mecânicas dos compósitos de Polipropileno pós-consumo reforçados com fibras de celulose Polímeros, 27, 84-90.

Suharty, N., Ismail, H., Diharjo, K., Handayani, D., \& Firdaus, M. (2016). Effect of Kenaf Fiber as a Reinforcement on the Tensile, Flexural Strength and Impact Toughness Properties of Recycled Polypropylene/Halloysite. Composites Procedia Chemistry, 19, $253-258$.

Szopik-Depczynska, K., Kedzierska-Szczepaniak, A., Szczepaniak, K., Cheba, K., Gajda, W., \& Ioppolo, G. (2018). Innovation in sustainable development: an investigation of the EU context using 2030 agenda indicators. Land Use Policy, 79, 251-262.

Todkar, S. S., Patil, S. A. (2019) Review on mechanical properties evaluation of pineapple leaf fibre (PALF) reinforced polymer composites. Composites Part B: Engineering, 174, 106927.

Triñanes, S., Rodriguez-Mier, P., Cobas, C., Sánchez, E., Phan-Tan-Luu, R., \& Cela, R. (2019). Robustness assessment in computer-assisted liquid chromatography procedures based on desirability functions. Journal of Chromatography, v.1609.

Candioti, V. L., Zan, M. M., Cámara, M. S., \& Goicoechea, H. C. (2014). Experimental design and multiple response optimization. Using the desirability function in analytical methods development. Talanta, 124, 123-138. 201.

Wanassi, B., Hariz, I. B., Ghimbeu, C. M., Vaulot, C., Hassen, M. B., \& Jeguirim, M. (2017). Carbonaceous adsorbents derived from textile cotton waste for the removal of Alizarin S dye from aqueous effluent: kinetic and equilibrium studies. Environmental Science and Pollution Research, $24,10041-10055$.

W D Callister (2012) Ciência e Engenharia de Materiais: Uma introdução. Chapman and Hall.

$\mathrm{Xu}, \mathrm{X}$. , Wang, Y., \& Tao, L. (2019). Comprehensive evaluation of sustainable development of regional construction industry in China. Journal of Cleaner Production, 211, 1078-1087.

Zeller, V., Towa, E., Degrez, M., \& Achten, W. M. J. (2018). Urban waste flows and their potential for a circular economy model at city-region level. Waste Management. 83, 83-94. 